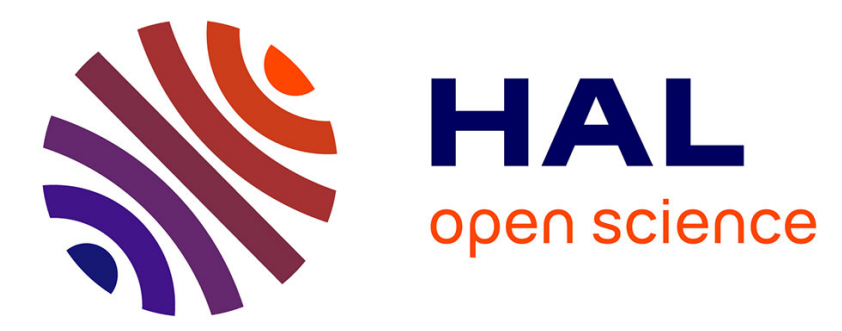

\title{
Bone ingrowth on the surface of endosseous implants. Part 2: Theoretical and numerical analysis
} Pedro Moreo, J.M. José Manuel García-Aznar, Manuel Doblaré

\section{To cite this version:}

Pedro Moreo, J.M. José Manuel García-Aznar, Manuel Doblaré. Bone ingrowth on the surface of endosseous implants. Part 2: Theoretical and numerical analysis. Journal of Theoretical Biology, 2009, 260 (1), pp.13. 10.1016/j.jtbi.2009.05.036 . hal-00554623

\section{HAL Id: hal-00554623 https://hal.science/hal-00554623}

Submitted on 11 Jan 2011

HAL is a multi-disciplinary open access archive for the deposit and dissemination of scientific research documents, whether they are published or not. The documents may come from teaching and research institutions in France or abroad, or from public or private research centers.
L'archive ouverte pluridisciplinaire HAL, est destinée au dépôt et à la diffusion de documents scientifiques de niveau recherche, publiés ou non, émanant des établissements d'enseignement et de recherche français ou étrangers, des laboratoires publics ou privés. 


\section{Author's Accepted Manuscript}

Bone ingrowth on the surface of endosseous implants.

Part 2: Theoretical and numerical analysis

Pedro Moreo, José Manuel García-Aznar, Manuel

Doblaré

PII:

S0022-5193(09)00246-X

DOI:

doi:10.1016/j.jtbi.2009.05.036

Reference:

YJTBI 5576

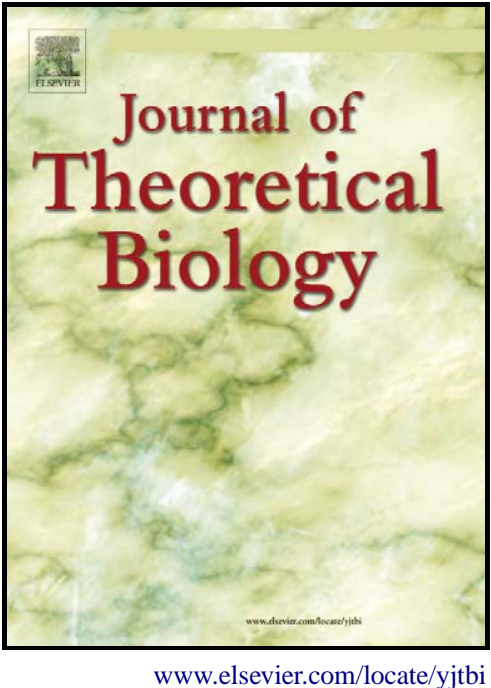

To appear in: $\quad$ Journal of Theoretical Biology

Received date: $\quad 14$ August 2008

Revised date: 21 May 2009

Accepted date: $\quad 24$ May 2009

Cite this article as: Pedro Moreo, José Manuel García-Aznar and Manuel Doblaré, Bone ingrowth on the surface of endosseous implants. Part 2: Theoretical and numerical analysis, Journal of Theoretical Biology, doi:10.1016/j.jtbi.2009.05.036

This is a PDF file of an unedited manuscript that has been accepted for publication. As a service to our customers we are providing this early version of the manuscript. The manuscript will undergo copyediting, typesetting, and review of the resulting galley proof before it is published in its final citable form. Please note that during the production process errors may be discovered which could affect the content, and all legal disclaimers that apply to the journal pertain. 


\title{
Bone ingrowth on the surface of endosseous implants. Part 2: theoretical and numerical analysis
}

\author{
Pedro Moreo, ${ }^{\text {a,b }}$ José Manuel García-Aznar, ${ }^{\text {a,c,* }}$ \\ Manuel Doblaré ${ }^{\mathrm{a}, \mathrm{c}}$

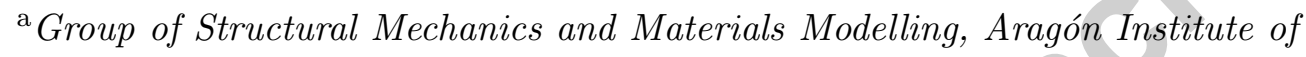 \\ Engineering Research (I3A), University of Zaragoza, Zaragoza, Spain \\ ${ }^{\mathrm{b}}$ EBERS Medical Technology S.L., Zaragoza, Spain \\ ${ }^{\mathrm{c}}$ CIBER-BBN Centro Investigación Biomédica en Red en Bioingeniería, \\ Biomateriales y Nanomedicina, Zaragoza, Spain
}

\begin{abstract}
The study of osseointegration of endosseous implants is a matter of great interest, mostly due to the increase in the use of many types of implants in clinical practice. Bone ingrowth results from a complex process, in which mechanics and biology play a major role. A wide variety of diverse factors can affect the development of the process, such as the properties or geometry of the implant surface, the mechanical stimulation or the initial cell conditions. In the first part of this article (Moreo et al., 2008) a model composed of a set of reaction-diffusion equations was proposed to simulate the formation of bone around implants, specially focused on the early stages of bone healing, that was able to contemplate the effects of surface microtopography. The goal of this second part is to use the model to analyse the effect
\end{abstract}


of factors such as cell stimulation, the initial cell concentration in the host bone and the geometry of the implant. For this purpose, two different simplified versions of the model are here analysed theoretically and further insight is gained from the study of the stability of fixed points and existence of travelling waves. Additionally, numerical simulations by means of the finite element method have been performed to examine the osseointegration of a dental implant with grooves at the surface of the threads. Results obtained from the analysis and simulations show that the model can reproduce some features of peri-implant bone ingrowth.

Key words: Osseointegration, Travelling wave, Groove, Finite element simulation

\section{Introduction}

In Part 1 of this work a model to study the formation of bone around endosseous implants was presented (Moreo et al., 2008). This model is composed by a set of coupled nonlinear reaction-diffusion equations, one for each of the eight variables of the model: three types of cells (platelets, osteogenic cells and osteoblasts), two generic types of growth factors (secreted by platelets and secreted by osteogenic cells and osteoblasts) and the volume fractions of the extracellular matrix constituents (fibrin network, woven inmature bone and lamellar mature bone).

Compared to current literature this work presented a novel aspect: it aimed to model the first biological phenomena that occur after implantation. In particular, it reproduces the activation of platelets after contacting the surface

\footnotetext{
* Corresponding author. Phone: (+34) 976761912 Fax: (+34) 976762578 E-mail address: jmgaraz@unizar.es (J.M. García-Aznar)
} 
of the implant. This activation depends on the level of plasma protein adsorption, which in turn is determined by the microtopography of the implant surface, among other factors (Park et al., 2001; Kikuchi et al., 2005). This scheme allows taking into account in a simple and efficient manner the effect of surface roughness on the whole osseointegration process. In fact, in Moreo et al. (2008) finite element simulations of bone deposition around a dental implant were performed and the model successfully reproduced the differences between contact and distance osteogenesis, which appear when the topography of the surface is changed from rough to polished (Berglundh et al., 2003; Davies, 2003).

The goal of the second part of this study is (i) to analyse the effect that the following factors have upon peri-implant bone ingrowth: initial cell conditions, implant geometry and different levels of cell stimulation; and (ii) to study how the composition of the matrix is altered as the ossification front propagates.

The first issue to be studied is the influence of the initial cell concentration at the surface of the host bone, that depends on the type of bone one considers. It is known that there are significant differences between cortical and trabecular bone. For example, cortical bone presents higher mechanical properties that trabecular bone, hence providing better primary stability for endosseous implants. However, trabecular bone presents other advantages, since the marrow that fills its pores is an excellent source of progenitor cells and contains a rich vasculature necessary for angiogenesis. Therefore, in order to fully evaluate the influence of the type of surrounding bone both mechanical and biological aspects have to be considered. Since this subject is rather complicated, we chose to focus on examining how the number of osteogenic cells at the surface of the host bone can drive the osteoconduction phase. 
Secondly, we evaluate how the implant geometry can affect the formation of bone, not only by changing the stresses and strains of the surrounding tissue, what has been experimentally verified (Vandamme et al., 2007), but also by altering certain biological phenomena, such as growth factor diffusion or the direction of cell migration.

Thirdly, we also study how the composition of the matrix is altered as the ossification front crosses a region which is affected by the kinetics of the different phenomena involved in the ossification process.

Finally, we also look into the effect of cell stimulation upon the global process of peri-implant bone healing.

To address all these points, two different methods are presented. On the one hand, finite element simulations of bone deposition around a dental implant are performed. On the other hand, two different simplified versions of the mathematical model are proposed and examined analytically. This second procedure is an attractive option, since it allows acquiring useful information about the qualitative behaviour of specific parts of the model. This would be more difficult to do through numerical simulation, given the complexity of the model and the large number of parameters involved.

Specifically, the analysis of the simplified models will consist in the determination of the existence and stability of fixed points and the appearance of travelling wave-like solutions. Travelling waves are particular solutions of reaction-diffusion systems, consisting in the propagation through the domain of a wave, which travels at constant speed with constant shape. They are a recurring topic in mathematical biology since there is a vast number of biological phenomena in which a key element seems to be the appearance of 
travelling waves (see, for example, Murray (2005)). Their analytical study is useful, because it provides information about the speed of propagation of the wave and the concentrations of the variable before and after the propagation. In peri-implant bone healing, the invasion of the cavity between an implant and the host bone by osteogenic cells and the subsequent propagation of an ossification front are two examples of travelling wave-like phenomena.

The article is organised as follows. In Section 2, the main features of the model are briefly discussed. In Sections 3 and 4, two different simplified versions of the model are proposed and studied analytically. Next, two-dimensional numerical simulations of bone ingrowth around a dental implant with grooves at the surface of the threads are presented in Section 5. Finally, a discussion on the results are given in Section 6.

\section{Mathematical modelling of bone ingrowth}

In this section we describe the most relevant characteristics of the mathematical model for peri-implant bone ingrowth that was developed in Part 1 of the paper (Moreo et al., 2008). In this model a continuum approach is adopted and therefore reaction-diffusion equations are used to compute the spatio-temporal evolution of the volumetric concentration of each specie.

Specifically, the model has eight variables, which can be classified into three groups:

Cell densities Three different types of cells are considered: platelets, osteogenic cells and osteoblasts, whose respective densities are denoted by $c, m$ and $b$. The inclusion of platelets in the model is noteworthy, since it 
allows taking into account early stages of bone healing and the influence of the implant surface microtopography. Osteogenic cells and osteoblasts are included, since they are known to be the two most important types of cells involved in peri-implant bone ingrowth (Davies, 2003). By osteogenic cells here we mean mesenchymal stem cells, which have the potential to differentiate into a variety of mesenchymal tissues, such as bone, cartilage, tendon, muscle, marrow, dermis and fat.

Growth factors A large number of growth factors and signalling molecules are known to intervene in the mediation of bone healing (Dimitriou et al., 2005). Hence, in order to obtain a tractable model some simplification has to be done. Since many growth factors have a similar effect in the process, it is possible to group them into families that have the same influence. In our case two generic families are distinguished: $s_{1}$ stands for the concentration of the growth factors secreted by platelets (PDGF, TGF- $\beta$ and others), which are known to stimulate the proliferation and migration of osteogenic cells (Kark et al., 2006); $s_{2}$ represents the concentration of osteogenic growth factors (BMPs, TGF- $\beta$ superfamily), which have a stimulating effect on the secretion of bone and on the proliferation of osteogenic cells (Linkhart et al., 1996).

Volume fractions of the matrix The extracellular matrix can be composed of three different constituents: first, the fibrin network, whose volume fraction is denoted by $v_{f}$, that is assumed to be the initial constituent of the matrix; second, woven or inmature bone, $v_{w}$, that is laid down by osteoblasts and, third, lamellar or mature bone, $v_{l}$, that comes from remodelling of woven bone.

Finally, the concentration of adsorbed proteins, $p$, appears in the formulation 
but is not a model variable but an input data, since its value is assumed to be known a priori as a function of the microtopography of the implant surface.

The model equations and a concise description of the biological interpretation of the model parameters can be found in the Appendix A. The reader is referred to Part 1 of the paper (Moreo et al., 2008) for a more thorough discussion on the model.

\section{Osteoconduction: analysis and simulation of a first simplified model}

The goal of this section is to examine in detail the phenomenon of osteoconduction and, in particular, analyse how this process can be influenced by the level of cell stimulation and the number of osteogenic cells available at the surface of the host bone. We recall that osteoconduction consists in the invasion of the cavity between the host bone and the implant by osteogenic cells. In fact, it is the most important phase in bone healing (Davies, 2003), since the following stages of bone formation and remodelling critically depend on the speed of migration of osteogenic cells and the level of cell density achieved after osteoconduction.

Within this section, we are only interested in osteoconduction. Therefore we focus exclusively on the equations that are involved in this process. With this in mind, a reduced version of the model was obtained. From this model we can extract analytical information regarding the existence of fixed points and travelling waves. Finally, the performance of numerical simulations of the simplified model permits to gain further insight into the dynamic behaviour 
of the model.

\subsection{Description of the simplified model}

The reduced model that we propose retains only the equations for two cellular types, osteogenic cells and osteoblasts, and one generic growth factor, $s_{2}$, secreted by both types of cells. Osteogenic cells must be necessarily taken into account, since they are the main cellular type involved in osteoconduction. Also, given that part of the osteogenic cells differentiate into osteoblasts as the cells move forward towards the surface of the implant, osteoblasts should also appear in the reduced model. Finally, the chemotactic effect of the generic growth factor $s_{2}$ upon the migration of osteogenic cells as well as the dependence of the rate of osteogenic cell proliferation upon $s_{2}$ motivate the inclusion of $s_{2}$ in the simplified model.

The nondimensionalised equations of the reduced model are:

$$
\begin{aligned}
\frac{\partial m}{\partial t} & =\nabla \cdot\left[D_{m} \nabla m-m B_{m 2} \nabla s_{2}\right]+ \\
\underbrace{\sigma\left(1+\frac{\alpha_{m} s_{2}}{\beta_{m}+s_{2}}\right)}_{f\left(m, s_{2}, b\right)} & \underbrace{m(1-m)-\left(\alpha_{m b}+A_{m}\right) m}_{g\left(m, s_{2}, b\right)} \\
\frac{\partial s_{2}}{\partial t} & =\nabla \cdot\left[D_{s 2} \nabla s_{2}\right]+\underbrace{\frac{\alpha_{2} s_{2}}{\beta_{2}+s_{2}}(m+b)-A_{s 2} s_{2}}_{h\left(m, s_{2}, b\right)}
\end{aligned}
$$

It is to be noted that the model equations have not been altered, since the flux and kinetic terms are the same as in the full model, neglecting the influence 
of the growth factor $s_{1}$. Only, for simplicity, the stimulating effect of $s_{2}$ on the secretion of $s_{2}$ by osteoblasts and osteogenic cells has been assumed to be the same for both cellular types. Also, note that given that this reduced model does not include platelets or the growth factor $s_{1}$, it is not suitable for the study of the effect of surface microtopography upon osteogenesis (Moreo et al., 2008). However, the general features of the osteoconduction phenomenon are independent of the type of osteogenesis, so still relevant information can be obtained from the reduced model.

\subsection{Existence and stability of homogeneous steady states}

We first examine the existence and stability of homogeneous steady states of the system (fixed points). Homogeneous steady states of the model will be denoted by $\mathbf{z}^{*}=\left(m^{*}, s_{2}^{*}, b^{*}\right)$ and verify $f\left(\mathbf{z}^{*}\right)=g\left(\mathbf{z}^{*}\right)=h\left(\mathbf{z}^{*}\right)=0$, where $f$, $g$ and $h$ are the reaction terms of Eqs. (1). Note that this simplified model is similar to the one proposed in Dale et al. (1995) and Olsen et al. (1996) for the study of wound healing disorders and presents equivalent mathematical properties. Here, however, we have performed a systematic study of all the possible travelling wave solutions that can appear depending on the parameter values and initial conditions.

The number and stability of the steady states and, therefore, the qualitative dynamical behaviour of the system, depend on the value of a bifurcation parameter $\chi$. As shown in the Appendix $B, \chi$ is a function of model parameters defined as $\chi=\alpha_{2}\left(1+\alpha_{m b} / A_{b}\right)$. Below we list all the possible homogeneous steady states that the system can exhibit (more details can be found in the Appendix B): 
Chronic non healing state $\mathbf{z}_{t}=(0,0,0)$ With no cells or growth factor, this steady state exists for all parameter values. Since bone deposition relies on the presence of osteoblasts, this state represents a situation of chronic absence of bone formation. This situation is of little relevance for the purposes of this paper and therefore this state will be forced to be unstable by imposing $\sigma>A_{m}+\alpha_{m b}$ (see Appendix B).

Low density state $\mathbf{z}_{0}=\left(m_{0}, 0, b_{0}\right)$ In this case the growth factor concentration is zero and the density of osteogenic cells and osteoblasts is $m_{0}=$ $1-\left(A_{m}+\alpha_{m b}\right) / \sigma$ and $b_{0}=m_{0} \alpha_{m b} / A_{b}$, respectively. Note that the density of cells is small compared to that of high density states $\mathbf{z}_{ \pm}$. This is a direct consequence of the absence of $s_{2}$, which leads to a low proliferation rate of osteogenic cells. Formation of new bone would certainly be possible in this situation, but at a low rate, since the velocity of bone matrix deposition depends on the concentration of osteoblasts, which in this case is small. This steady state exists with realistic (positive) values for $m_{0}$ and $b_{0}$ if and only if $\sigma>A_{m}+\alpha_{m b}$, what was assumed as a global condition in the previous paragraph, and is stable if and only if $0<\chi<\chi^{(2)}=A_{s 2} \beta_{2} / m_{0}$.

High density states $\mathbf{z}_{ \pm}=\left(m_{ \pm}, s_{2 \pm}, b_{ \pm}\right)$This pair of steady states only exists when $\chi>\chi^{(1)}\left(\right.$ where $\left.\chi^{(1)}<\chi^{(2)}\right)$. The analytical expressions for $\chi^{(1)}$ and $\mathbf{z}_{ \pm}$are not simple and can be found in Appendix B. In this case it is possible to show that $\mathbf{z}_{+}$is stable whereas $\mathbf{z}_{-}$is unstable. However, when $\chi>\chi^{(2)}, \mathbf{z}_{0}$ becomes unstable, $\mathbf{z}_{-}$takes negative values and hence only $\mathbf{z}_{+}$ is biologically relevant. Theoretically, the stable state $\mathbf{z}_{+}$is attractive since high values of the osteoblast density and the growth factor concentration ensure a high rate of bone matrix deposition. These results are summarised qualitatively in Fig. 1. 


\subsection{Biophysical interpretation of $\chi$}

It is clear that the number of homogeneous steady states, as well as their stability can be determined in terms of the bifurcation parameter $\chi$, which has a direct biophysical interpretation. Note that $\chi$ is a monotonically increasing function of the rate of growth factor secretion, $\alpha_{2}$, and the rate of cell differentiation, $\alpha_{m b}$. These two cellular processes take place at a faster rate when cells are highly stimulated. Hence, $\chi$ can be seen as a measure of the general level of cell stimulation, irrespective of the actual nature of the stimulus (chemical, mechanical, etc.).

Thus, the qualitative dynamical behaviour of the system can be classified into three different regimes depending on the value of $\chi$, assuming that the rest of parameters are kept constant (see Fig. 1):

Regime I: $\chi \in\left(0, \chi^{(1)}\right)$ In this situation the system will tend to evolve to $\mathbf{z}_{0}$, since it is globally stable. However, given the reduced number of cells existing at $\mathbf{z}_{0}$, new bone formation will take place at a slow rate. Theoretically, this condition could correspond to a slow implant osseointegration, due to an insufficient stimulation.

Regime II: $\chi \in\left(\chi^{(1)}, \chi^{(2)}\right)$ In this case two locally stable states exist, $\mathbf{z}_{0}$ and $\mathbf{z}_{+}$, each one with its own region of attraction. Depending on the initial or boundary conditions, the system will tend to move to one of the two stable states. From the point of view of peri-implant bone healing, it is very desirable that the system evolves towards $\mathbf{z}_{+}$, since this state offers a large number of osteoblasts and a high concentration of $s_{2}$, what guarantees fast bone deposition. Mathematically, the transition from regime I to II 
corresponds to a saddle-node bifurcation (Strogatz, 2001), leading to the appearance of $\mathbf{z}_{ \pm}$.

Regime III: $\chi \in\left(\chi^{(2)}, \infty\right)$ Here $\mathbf{z}_{+}$is the stable state and corresponds to the well-known situation in which an adequate stimulation enhances bone ingrowth and osseointegration of the implant. The transition to this third regime from the second one takes place now through a transcritical bifurcation (Strogatz, 2001).
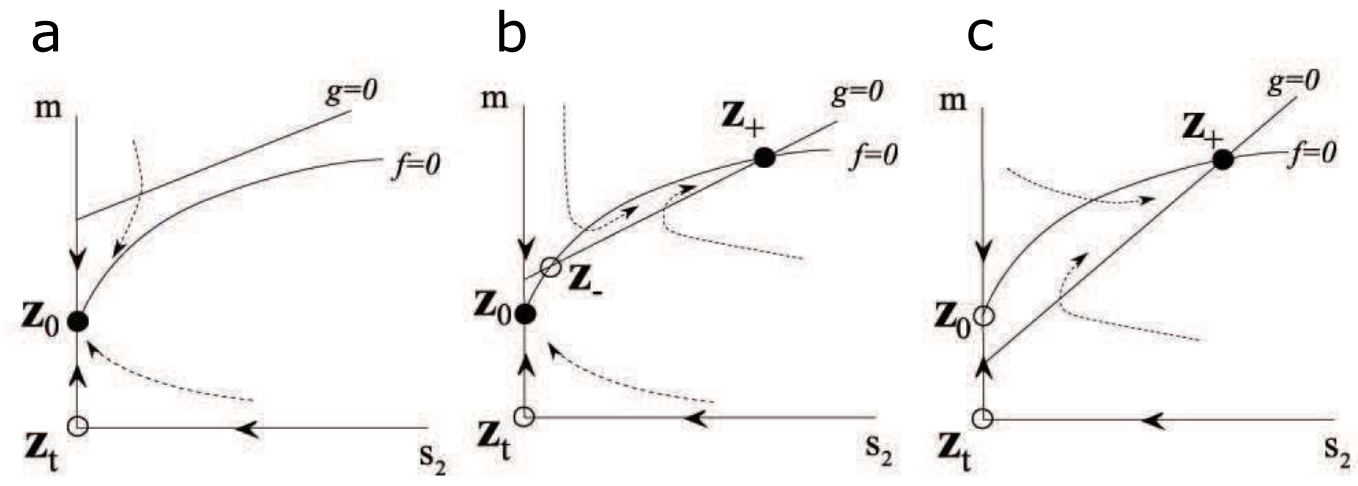

Figure 1. Phase space diagrams of the simplified model in the $m-s_{2}$ plane. Biologically realistic steady states are depicted with circles, filled in the stable case and void in the unstable one. (a) Regime I: $\chi \in\left(0, \chi^{(1)}\right)$, (b) Regime II: $\chi \in\left(\chi^{(1)}, \chi^{(2)}\right)$, (c) Regime III: $\chi \in\left(\chi^{(2)}, \infty\right)$.

\subsection{Travelling wave solutions}

During the osteoconduction phase, the space between the implant and the host bone is invaded by osteogenic cells that migrate from the bone. Mathematically, this can be seen as the appearance a travelling wave solution of the system (1). Thus, the study of travelling waves with our reduced system is interesting, since it permits to extract information with a direct biological interpretation, namely: 
- Bounds for the speed of propagation of the front. This speed is a measure of the velocity of invasion of the space around the implant by osteogenic cells. Therefore, it is a critical parameter that conditions the time needed for the implant to osseointegrate.

- Steady states between which travelling waves will develop. Again, this constitutes a relevant source of information, since it allows us to know the concentrations of cells and the growth factor before and after the propagation of the wave. In our case, the density of cells after the propagation of the wave is another critical value for osseointegration, given that the larger the density of cells, the faster bone matrix will be deposited in subsequent phases.

Formally travelling waves are solutions of the form $\mathbf{z}(x, t)=\mathbf{Z}(r)$, where $r=$ $x-a t$ and $a$ is the wave velocity (see Murray (2005) for a detailed description of travelling wave analysis). Below, we present the different travelling waves that can develop depending on the regime that we consider (Appendix $\mathrm{C}$ describes the mathematical analysis):

Regime I: $\chi \in\left(0, \chi^{(1)}\right)$ It can be shown that in this interval a travelling wave from $\mathbf{z}_{t}$ to $\mathbf{z}_{0}$ does exist. A lower bound for the wave speed is found analytically, namely $a \geq a_{\min }^{(1)}=2 \sqrt{D_{m}\left[\sigma-\left(\alpha_{m b}+A_{m}\right)\right]}$. In the simulations shown in the next section, it is found that the numerically evaluated wave speed compares well with this minimum value $a_{\mathrm{min}}^{(1)}$. This also holds true for the rest of travelling waves described below.

Regime II: $\chi \in\left(\chi^{(1)}, \chi^{(2)}\right)$ The same travelling wave from $\mathbf{z}_{t}$ to $\mathbf{z}_{0}$ also appears in this regime, with the same bound for the wave speed. Further, a second travelling wave joining $\mathbf{z}_{0}$ with $\mathbf{z}_{+}$can also develop, with no analytic lower bound for the wave velocity. Note that there is no travelling wave 
solution joining directly the states $\mathbf{z}_{t}$ and $\mathbf{z}_{+}$.

Regime III: $\chi \in\left(\chi^{(2)}, \infty\right)$ The number of possibilities is greater in this interval. In first place, a travelling wave solution from $\mathbf{z}_{t}$ to $\mathbf{z}_{0}$ also exists, now with the condition for the wave speed $a \geq \max \left\{a_{\min }^{(1)}, a_{\min }^{(2)}\right\}$, where

$$
a_{\mathrm{min}}^{(2)}=2 \sqrt{D_{s}\left(\frac{\chi m_{0}}{\beta_{2}}-A_{s 2}\right)}
$$

Next, the travelling wave joining $\mathbf{z}_{0}$ with $\mathbf{z}_{+}$also appears, in this case with the restriction for the wave speed $a \geq a_{\min }^{(2)}$. Finally, the steady states $\mathbf{z}_{t}$ and $\mathbf{z}_{+}$are now connected by a third travelling wave type solution, with a lower bound for the velocity equal to $a_{\mathrm{min}}^{(1)}$.

\subsection{Numerical simulation of the simplified model}

Armed with the knowledge of the main features of the simplified model thanks to the mathematical analysis described above, we now investigate numerically the formation of travelling wavefronts depending on cell stimulation, characterised in this approach by $\chi$. For this purpose, the nondimensionalised system described by Eqs. (1) has been solved using the MATLAB solver pdepe in the one dimensional domain $x \in[0,100]$, where $x$ is the nondimensional space variable. This domain represents the space between the host bone and the implant. Hence, the left end stands for the surface of the bone while the right one stands for the surface of the implant. As stated in previous sections, with this simplified model we are able to reproduce only the osteoconduction phase. The initial conditions must therefore account for the situation before this phase has started, that is, no osteoblasts, a low concentration of growth factor $s_{2}$ in the whole domain and a low concentration of osteogenic cells near the surface of the host bone and null in the rest. Zero flux boundary conditions 
have been considered at both ends, representing the impossibility of cells and growth factors to migrate through the surface of the implant and the bone. The results of the numerical simulations with different initial conditions and three different sets of parameter values $\chi=1.5,3.0,7.5$, corresponding to the three different regimes of the model, are shown in Figs. 2-6.
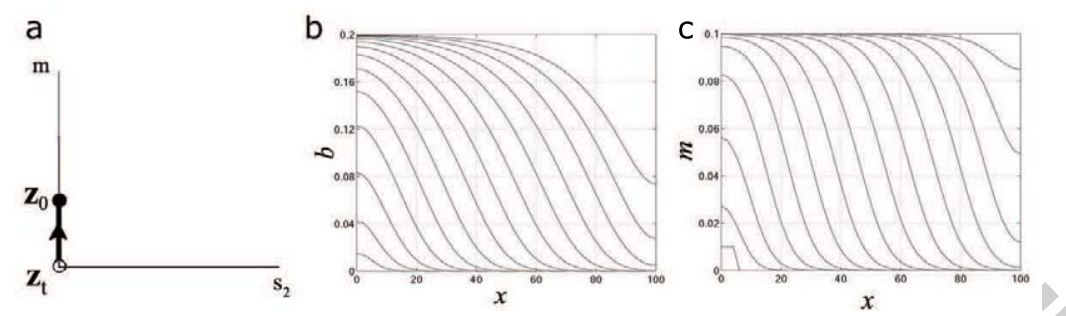

Figure 2. Numerical solution of the model given by Eqs. (1) in the Regime I $(\chi=1.5)$ with the following initial conditions $\left(m(x, 0)=m_{+} / 10\right.$ if $x<5,0$ otherwise; $\left.s_{2}(x, 0)=s_{2+} / 100 ; b(x, 0)=0\right)$. (a) Phase portrait with the trajectory of the travelling wave. Nondimensionalised values of (b) osteoblast concentration $b$ and (c) osteogenic cells concentration $\mathrm{m}$. Growth factor concentration $s_{2}$ is not shown since it decreases to zero within the first increments of the simulation and remains constant from that moment. Wavefronts travel from left (surface of the host bone) towards the right (surface of the implant) at constant speed. Solutions are shown at successive time intervals $\tau=0,10,20, \ldots, 150$. Nondimensional parameters are as follows: $D_{m}=D_{s 2}=1.0, B_{m 2}=0, \sigma=1.5, \alpha_{m}=\beta_{m}=2.0, A_{m}=1.25, \alpha_{m b}=0.1$, $A_{s 2}=0.5, \alpha_{m 2}=0.5, \beta_{m 2}=1.0, A_{b}=0.05$. Critical values of bifurcation parameter $\chi$ are $\chi^{(1)} \simeq 2.08$ and $\chi^{(2)}=5.0$. All the parameters except $\alpha_{m 2}$ take the same value in Figs. 2-6.

For the set of parameters chosen in Fig. 2, the system lies in the Regime I. After an initial transient period, wavefronts for $m$ and $b$ develop, acquire constant shape and move along the domain with constant speed towards the right, that is, from the host bone towards the implant surface. This corresponds to a travelling wave from the unstable steady state $\mathbf{z}_{t}$ to the stable steady state 

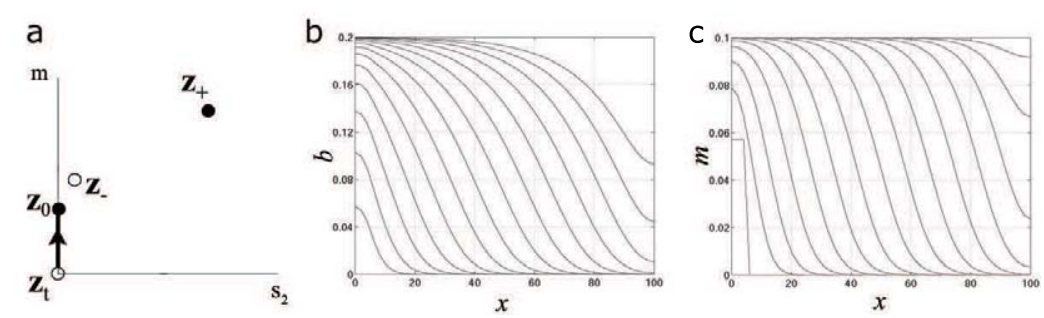

Figure 3. Numerical solution of the model given by Eqs. (1) in the Regime II $\left(m(x, 0)=m_{+} / 10\right.$ if $x<5,0$ otherwise; $\left.s_{2}(x, 0)=s_{2+} / 100 ; b(x, 0)=0\right)$. (a) Phase portrait with the trajectory of the travelling wave. Nondimensionalised values of (b) osteoblast concentration $b$ and (c) osteogenic cells concentration $m$. Growth factor concentration $s_{2}$ is not shown since it decreases to zero within the first increments of the simulation and remains constant from that moment. Wavefronts travel from left (surface of the host bone) towards the right (surface of the implant) at constant speed. Solutions are shown at successive time intervals $\tau=0,10,20, \ldots, 150$.

$\mathbf{z}_{0}$. Note that the concentration of the growth factor rapidly tends to zero and remains there during the rest of the simulation. This could be expected since this travelling wave lies in the $s_{2}=0$ plane in the phase diagram (see Fig. 1a). In a biological context, this represents the invasion of the cavity between bone and implant by cells, that is, the osteoconduction phase.

With equivalent initial conditions, a very similar result is obtained in the Regime II and is shown in Fig. 3. However, with the same parameter values as in Fig. 3, if the initial conditions at one of the ends of the domain - the host bone end-, are in the attraction region of the steady state $\mathbf{z}_{+}-$a high density of osteogenic cells-, the behaviour of the solution is markedly different, as can be seen in Fig. 4. In this case we impose as initial condition a high concentration both of osteogenic cells and growth factor in the neighbourhood of the left end. This causes the formation of a first wavefront between $\mathbf{z}_{t}$ and $\mathbf{z}_{0}$ and, immediately later, a second one between $\mathbf{z}_{0}$ and the high cell 

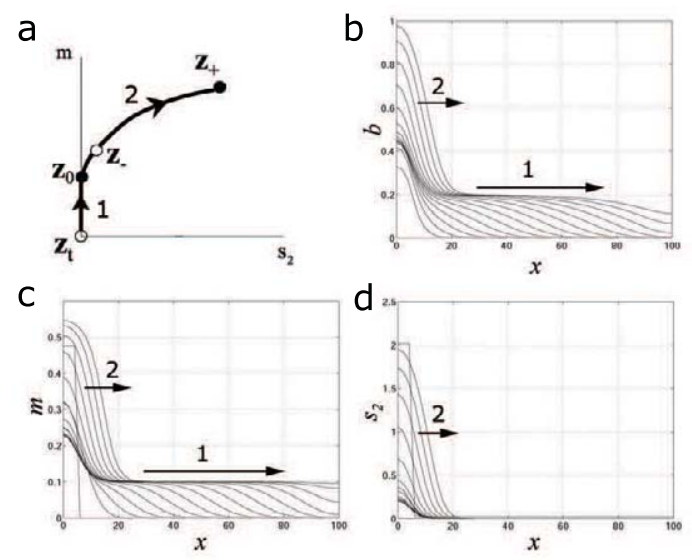

Figure 4. Numerical solution of the model given by Eqs. (1) in the Regime II $\left(\chi=3.0, \alpha_{m 2}=1.0\right)$ with the following initial conditions $\left(m(x, 0)=m_{+} / 1.2\right.$ if $x<5,0$ otherwise; $\left.s_{2}(x, 0)=s_{2+} / 1.2 ; b(x, 0)=0\right)$. (a) Phase portrait with the trajectory of the travelling waves. Nondimensionalised values of (b) osteoblast concentration $b,(\mathrm{c})$ osteogenic cells concentration $m$ and (d) growth factor concentration $s_{2}$. Two travelling fronts develop, one from $\mathbf{z}_{t}$ to $\mathbf{z}_{0}$, highlighted with arrow 1 , and next, after the first one has fully developed, a second one from $\mathbf{z}_{0}$ to $\mathbf{z}_{+}$, highlighted with arrow 2 . Solutions are shown at successive time intervals $\tau=0,10,20, \ldots, 150$.

concentration state $\mathbf{z}_{+}$. Biologically this also represents the characteristic cell migration of the osteoconduction phase, but now leading to a higher cell density compared to the results of Figs. 2-3. Note that the second wave speed is much slower compared to the first one, so the desirable final state $\mathbf{z}_{+}$is reached after a long healing period. Note also that the development of a single travelling wave between $\mathbf{z}_{t}$ and $\mathbf{z}_{+}$is not possible, since this trajectory does not exist in the phase diagram of this regime. The osseointegration velocity is therefore limited in this case by the small second wave speed that does not have any analytical lower bound.

The most desirable situation is shown in Fig. 5, corresponding to a direct and fast travelling wave between $\mathbf{z}_{t}$ and $\mathbf{z}_{+}$. This is possible because the system is 

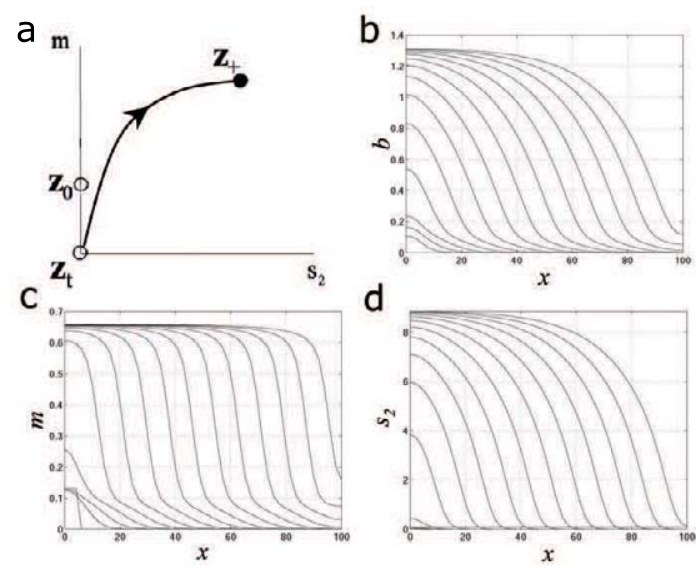

Figure 5. Numerical solution of the model given by Eqs. (1) in the Regime III $\left(\chi=7.5, \alpha_{m 2}=2.5\right)$ with the following initial conditions $\left(m(x, 0)=m_{+} / 5\right.$ if $x<5$, 0 otherwise; $s_{2}(x, 0)=s_{2+} / 100 ; b(x, 0)=0$ ). (a) Phase portrait with the trajectory of the travelling wave. Nondimensionalised values of (b) osteoblast concentration $b$, (c) osteogenic cells concentration $m$ and (d) growth factor concentration $s_{2}$. Wavefronts travel from left (surface of the host bone) towards the right (surface of the implant) at constant speed. Solutions are shown at successive time intervals $\tau=0,10,20, \ldots, 150$.

now in the Regime III, where $\mathbf{z}_{0}$ and $\mathbf{z}_{+}$do not compete for regions of attraction since $\mathbf{z}_{0}$ is now unstable (Fig. 1c), and also because the initial concentration of osteogenic cells at the end of the host bone is relatively high. Otherwise a two-wavefront-type solution would emerge again, as is shown in Fig. 6. In this latter case, after the propagation of the first wavefront, the solution in the whole domain corresponds to the unstable state $\mathbf{z}_{0}$, so spontaneously a second travelling wave leading to the invasion of the domain by a high cell density is formed. Note that this solution, although slower than the one of Fig. 5, is still attractive, since it is considerable faster than the invasion in Fig. 4 and does not require initial conditions close to $\mathbf{z}_{+}$. Hence, in this case the adequate cell stimulation compensates the poor source of osteogenic cells. 

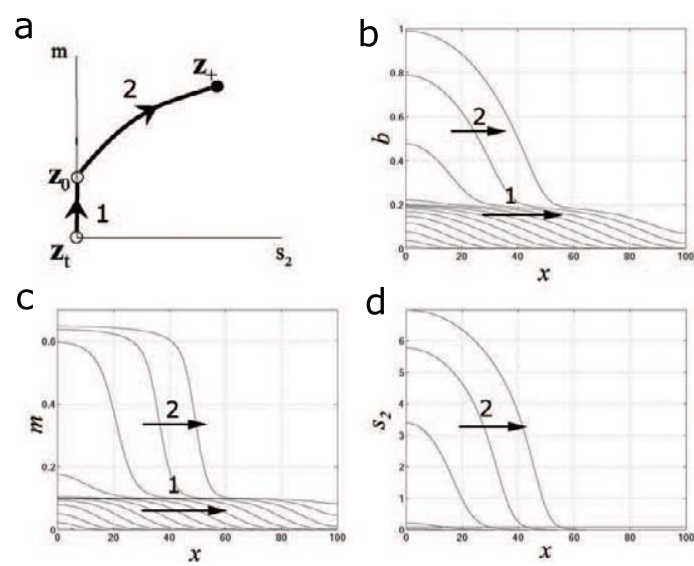

Figure 6. Numerical solution of the model given by Eqs. (1) in the Regime III $\left(\chi=7.5, \alpha_{m 2}=2.5\right)$ with the following initial conditions $\left(m(x, 0)=m_{+} / 10\right.$ if $x<5$, 0 otherwise; $s_{2}(x, 0)=s_{2+} / 100 ; b(x, 0)=0$ ). (a) Phase portrait with the trajectory of the travelling waves. Nondimensionalised values of (b) osteoblast concentration $b,(\mathrm{c})$ osteogenic cells concentration $m$ and (d) growth factor concentration $s_{2}$. Two travelling fronts develop, one from $\mathbf{z}_{t}$ to $\mathbf{z}_{0}$, highlighted with arrow 1 , and next, after the first one has fully developed, a second one from $\mathbf{z}_{0}$ to $\mathbf{z}_{+}$, highlighted with arrow 2. Solutions are shown at successive time intervals $\tau=0,10,20, \ldots, 150$.

\section{Propagation of the ossification front: a second simplified model}

In the previous section the volume fractions of the components of the matrix were not taken into account so we could not examine the new bone formation. Here, we propose another simplified model in which growth factors and platelets are neglected with the purpose of exploring the shape of the ossification front. From the experiments it is well known that either the implant becomes surrounded by an ossification front coming from the host bone (distance osteogenesis) or new bone forms first on the implant surface and the front propagates from there towards the inside of the cavity between the host bone and the implant (contact osteogenesis). The aim of this section is to study how the composition of the matrix is altered as the ossification front 
crosses a region of the cavity, independently of the type of osteogenesis that we consider.

To study the deposition of bone, it is not necessary to contemplate the equations for platelets and the growth factor $s_{1}$, because the effect of platelet activation is to alter the direction of propagation of the ossification front (contact/distance osteogenesis), but not the way new bone is laid down as the front propagates. Furthermore, the growth factor $s_{2}$ needs not to be included in such a model, since its main effect is to stimulate osteogenesis by increasing the rate at which bone formation takes place, but again does not provoke any substantial change in the way bone matrix is deposited.

Therefore the reduced model that we suggest retains the equations for the osteogenic cells, $m$, and osteoblasts, $b$, as well as the volume fractions of the components of the matrix: fibrin network, $v_{f}$, woven bone, $v_{w}$, and lamellar bone, $v_{l}$. The nondimensionalised equations of the reduced model read as:

$$
\begin{aligned}
\frac{\partial m}{\partial t} & =D_{m} \nabla^{2} m+\sigma m(1-m)-\left(\alpha_{m b}+A_{m}\right) m \\
\frac{\partial b}{\partial t} & =\alpha_{m b} m-A_{b} b \\
\frac{\partial v_{f}}{\partial t} & =-\alpha_{w} b v_{f}\left(1-v_{w}\right) \\
\frac{\partial v_{w}}{\partial t} & =\alpha_{w} b v_{f}\left(1-v_{w}\right)-\gamma v_{w}\left(1-v_{l}\right) \\
\frac{\partial v_{l}}{\partial t} & =\gamma v_{w}\left(1-v_{l}\right)
\end{aligned}
$$

The flux and kinetic terms are straightforwardly inferred from the full model, simply neglecting the influence of the growth factors. The kinetics of the sys- 
tem is now simpler than in Section 3, so the analysis is simplified. We follow the same steps as before: first the fixed points of the system are determined, then the possibility of having travelling wave solutions is studied analytically and finally one dimensional finite difference simulations are performed to examine the shape of the wave fronts.

Denoting the homogeneous steady states by $\mathbf{z}^{*}=\left(m^{*}, b^{*}, v_{f}^{*}, v_{w}^{*}, v_{l}^{*}\right)$, we find two of them of interest: $\mathbf{z}_{0}^{*}=\left(m_{0}, b_{0}, 0,0,1\right)$ and $\mathbf{z}_{t}^{*}=(0,0,1,0,0)$, where $m_{0}=1-\left(\alpha_{m b}+A_{m}\right) / \sigma$ and $b_{0}=\alpha m_{0} / A_{b}$. We assume that $\sigma>\alpha_{m b}+A_{m}$ so that $m_{0}$ is positive and physically realistic. The first state, $\mathbf{z}_{0}$, represents a situation in which the composition of the matrix corresponds to pure lamellar bone. It is therefore the result of the whole bone healing process. On the other hand, $\mathbf{z}_{t}$ stands for the situation that appears just after implantation, where the extracellular matrix is composed only of a fibrin network and there are no osteogenic cells or osteoblasts. Using standard techniques (Murray, 2005) it is possible to show that a travelling wave can develop between $\mathbf{z}_{t}$ and $\mathbf{z}_{0}$ with minimum wave speed of $2 \sqrt{D_{m}\left[\sigma-\left(\alpha_{m b}+A_{m}\right)\right]}$. After the propagation of this wave the composition of the matrix changes from a pure fibrin network to lamellar bone. The spatial propagation of this travelling wave solution is interpreted biologically as the advance of the ossification front.

Following the same procedure as in the previous section, we solve the system of Eqs. (3) numerically in a one dimensional domain $x \in[0,100]$ that can be thought as the cavity between the implant and the host bone. Again the left end of the domain represents the surface of bone whereas the right one stands for the surface of the implant. Zero flux boundary conditions are applied to both ends. We model the propagation of the ossification front in a case of distance osteogenesis, so the initial conditions consist of a low concentration 
of osteogenic cells near the surface of bone and null in the rest of the domain, a null concentration of osteoblasts and a matrix composed exclusively by fibrin. The results of the numerical simulation are shown in Figs. 7-8.

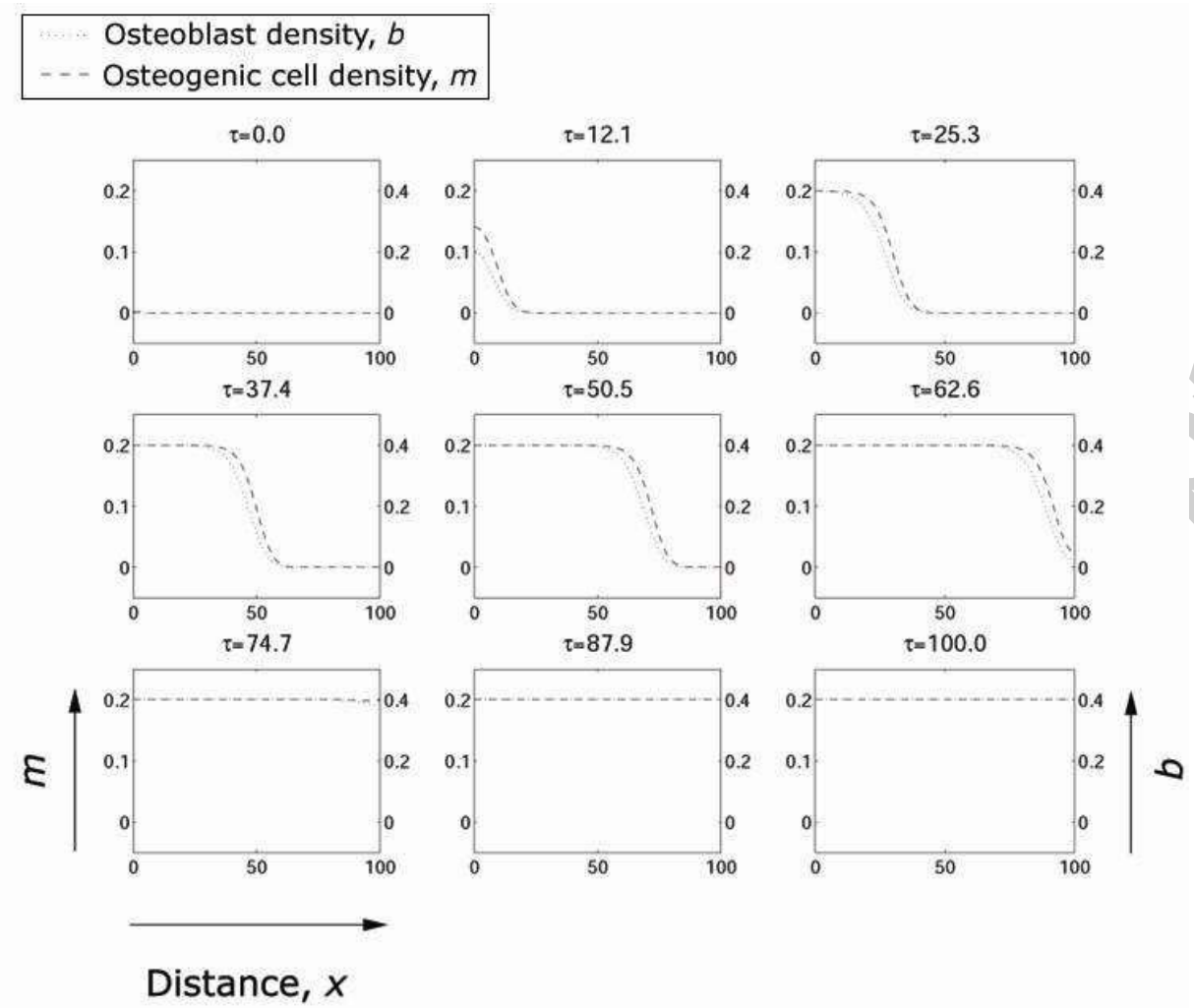

Figure 7. Evolution of the density of osteogenic cells, $m$, and osteoblasts, $b$. Wavefronts travel from left (host bone) to right (implant) at constant speed. $x$ denotes the distance to the surface of the host bone. Nondimensional parameters are as follows: $D_{m}=1.0, \sigma=2.5, A_{m}=1.25, \alpha_{m b}=1.0, A_{b}=0.5, \alpha_{w}=1.0, \gamma=0.25$.

In the figures we appreciate the early formation, development up to a constant shape and propagation at a constant velocity of the ossification front. Observe the shape of the cellular waves (Fig. 7): they are very similar, although the osteoblast wave is slightly delayed with respect to the one of osteogenic cells. This is obvious since the only source of osteoblasts is precisely the differentiation from the osteogenic precursor. One might intuitively expect a greater delay between the two moving fronts of cells, but this result is realistic if one 


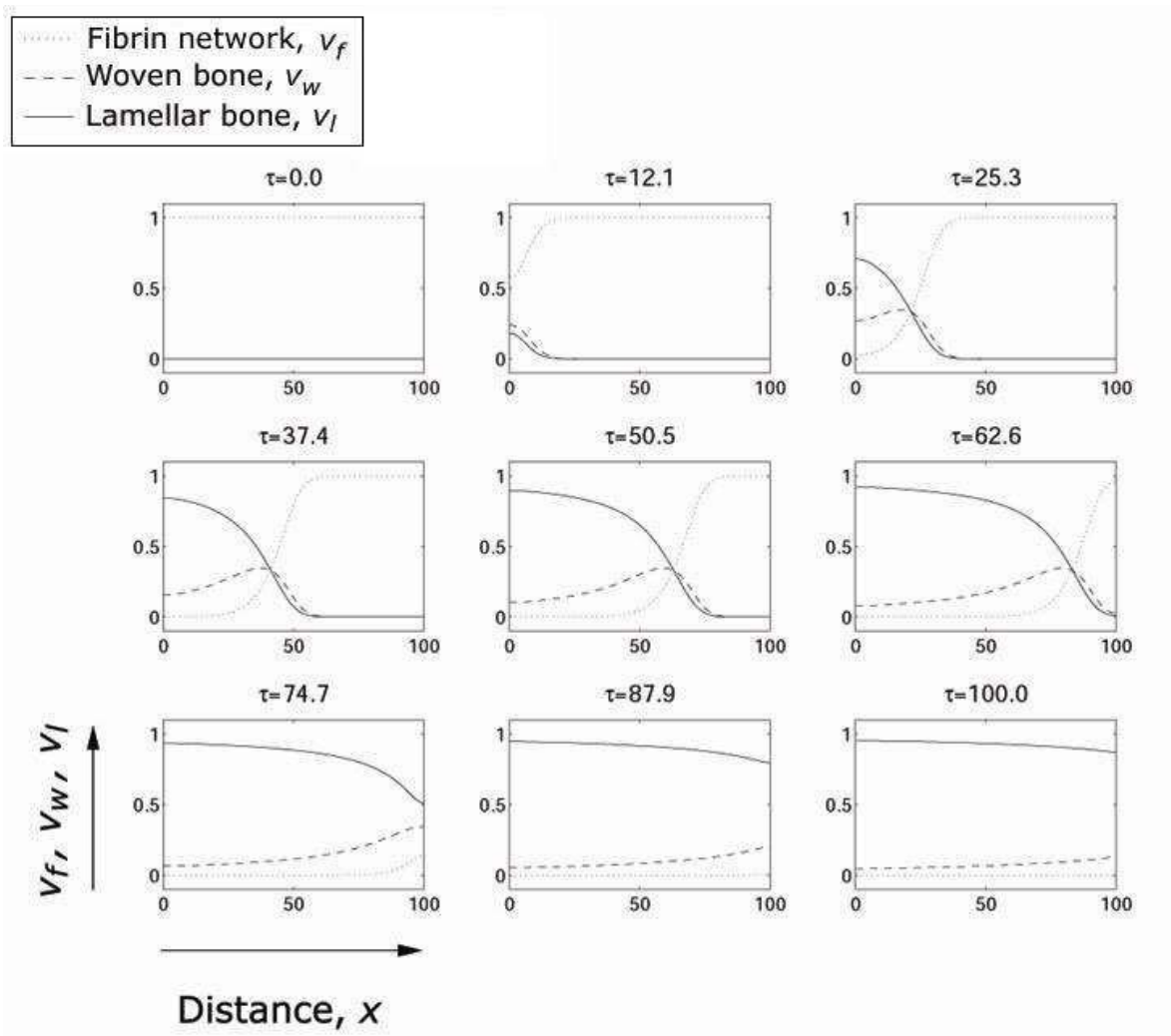

Figure 8. Evolution of the composition of the matrix: volume fractions of fibrin, $v_{f}$, woven bone, $v_{w}$ and lamellar bone, $v_{l}$. Wavefronts travel from left (surface of the host bone) towards the right (surface of the implant) at constant speed. $x$ denotes the distance to the surface of the host bone.

considers that cell differentiation is a fast process (Bailón-Plaza and van der Meulen, 2001) compared to the time scale of the full invasion of the cavity by bone.

Note that the profiles of the volume fractions along the ossification front have a very particular shape. In first place, ahead of the front (right side in Fig. 8) the composition of the matrix corresponds to pure fibrin. Far behind the front (on the left side), the composition corresponds to lamellar bone. This is an area that has already been crossed by the ossification front. Between these areas there is a transition region where the volume fraction of woven bone reaches 
a maximum. Hence, the profile of woven bone corresponds to a propagating pulse which is notably asymmetric, since the ahead (right) front is markedly steeper than the rear (left) one. This is again in agreement with the basis of bone healing since the processes that take place in the head front of the pulse (cell differentiation, laying done of bone matrix) are quite fast compared to the speed of remodelling, that occurs at the rear tail of the pulse. Observe that at a certain instant of time deposition of bone is localised in the small region in the front part of the wave.

\section{Influence of geometry: numerical simulation of the full model}

\subsection{Description of the simulation}

In Part 1 of this paper (Moreo et al., 2008) the complete model was numerically solved by means of the finite element method in a geometry corresponding to the cavity between two threads of a dental implant and host bone in which the implant was placed. The simulations were performed with two different implant surface microtopographies to show the ability of the model to reproduce the differences between contact and distance osteogenesis. In this section we present the results of numerical simulations of bone ingrowth around a screw-shaped dental implant characterised by threads with inclined walls and longitudinal grooves of circular section, proposed by some manufactures as an innovative way to enhance the formation of new bone and achieve a better interlock between bone and implant (Hall et al., 2005). We intend to investigate the effect of these grooves and find out if the model provides any clue about the role of this particular implant geometry in peri-implant bone formation. 
All the details of the simulation have already been described in detail in Moreo et al. (2008) and are here briefly discussed. The computational domain is two dimensional and is depicted in Fig. 9a, where the grooves of the surface of the implant thread can be seen. Two different values of the density of adsorbed proteins at the implant surface have been considered: $p=0.5 \mu \mathrm{g} / \mathrm{mm}^{-2}$, simulating the case of a high microtopography implant, and $p=0.1 \mu \mathrm{g} / \mathrm{mm}^{-2}$, in the case of an implant of low surface microtopography (Sela et al., 2007).

As initial conditions, we have considered a concentration of $2.5 \times 10^{8}$ platelets $/ \mathrm{ml}$, being this high value characteristic of blood (Ganong, 2005), and residual concentrations of osteogenic cells and osteoblasts of $10^{3}$ cells $/ \mathrm{ml}$. Both growth factors are also present at the initial time at a concentration of $1 \mathrm{ng} / \mathrm{ml}$, residual in comparison with typical values in bone healing (Joyce et al., 1990). Zero flux boundary conditions have been applied for all the species of model at the surface of the implant and the host bone, except for the concentration of osteogenic cells at the surface of bone during the first 14 days, that was fixed to $2 \times 10^{5}$ cells $/ \mathrm{ml}$. The healing period that was simulated consisted of twelve months, more than sufficient in clinical practice to obtain a full osseointegration of the implant and achieve a high degree of remodelling of woven bone into lamellar bone. Details on the numerical method and the values of the model parameters can be found in Moreo et al. (2008).

\subsection{Results}

In first place, note that as early as four days after implantation some differences in the concentration of the growth factor $s_{1}$ secreted by platelets appear between the the two types of microtopographies. In the rough implant (high 


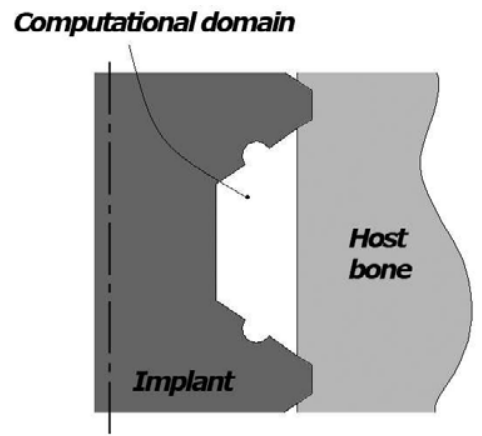

(a)

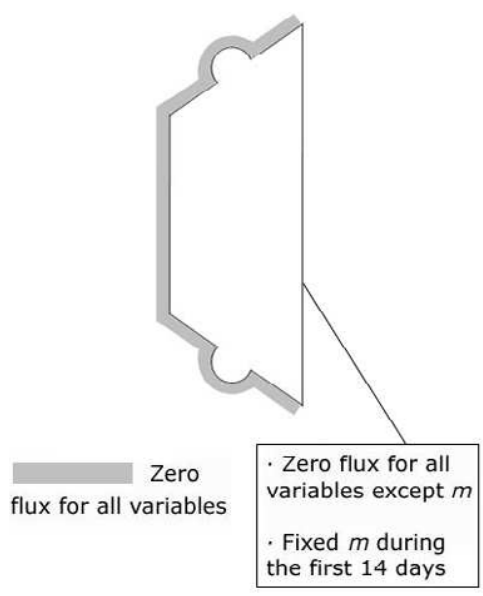

(b)

Figure 9. (a) Sketch of the insertion of a screw-shaped dental implant in a drilled cavity of bone, where the computational domain of our simulations is highlighted. (b) Boundary conditions.

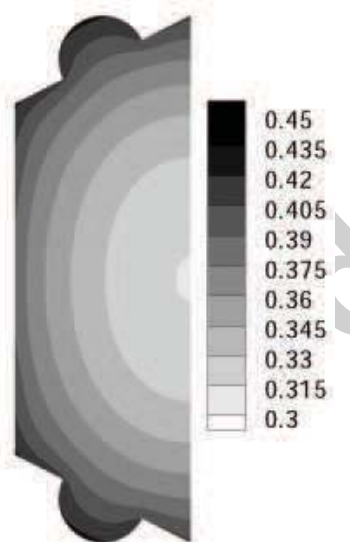

(a)

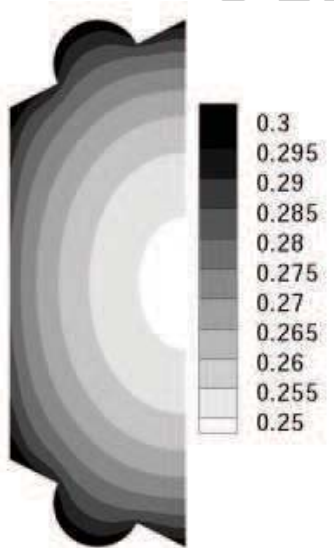

(b)

Figure 10. Concentration of growth factor $s_{1}(\mathrm{x} 100 \mathrm{ng} / \mathrm{ml})$ after four days in the case of an implant with (a) high microtopography and (b) low microtopography. microtopography) $s_{1}$ reaches a value $\sim 50 \%$ higher than in the polished one (low microtopography), and additionally a marked gradient of the concentration develops, being higher near the implant surface. However, more important than this discrepancy, originated from the different types of surfaces and already detected in Part 1 of this work (Moreo et al., 2008), is the effect of the geometry of the grooves upon the concentration of $s_{1}$. Note that the highest 
value of $s_{1}$ is reached near the implant surface inside the grooves and it also there where a more abrupt gradient in the concentration is obtained. This could be expected just by the geometry of the surface: the greatest rate of growth factor secretion appears next to the surface, where platelets are activated thanks to the presence of adsorbed proteins. Thus, $s_{1}$ diffuses from the surface towards the internal regions of cavity, but the curvature of the surface at the grooves is responsible for a concentrating effect, so the growth factor tends to accumulate inside the grooves. This strongly conditions the posterior behaviour of osteogenic cells in several ways. In first place, the migration of osteogenic cells is affected by chemotaxis, so they preferentially move along gradients of the growth factor, that is, inside the grooves. Furthermore, the rate of cell mitosis and differentiation is also enhanced by the local level of $s_{1}$. Therefore, a higher density of osteogenic cells is achieved inside the grooves, particularly in the case of the high microtopography surface implant, where the effect of grooves is more pronounced. Moreover, these cells differentiate into the osteoblastic phenotype at a faster rate.

The characteristic effect of this altered migration, proliferation and differentiation of cells inside the grooves can be noticed in the formation of bone. In Figs. 11 and 12 the temporal evolution of the volume fraction of lamellar bone has been represented for a rough and a polished implant, respectively. First, remark that again the characteristic contact osteogenesis with initial formation of lamellar bone on the implant surface occurs with the rough implant, while with the polished implant distance osteogenesis predominates and a well-defined ossification front propagates in this case from the surface of the surrounding host bone inside the cavity. This was presented in Part 1 of the work (Moreo et al., 2008). Nevertheless, what must be here highlighted is the 


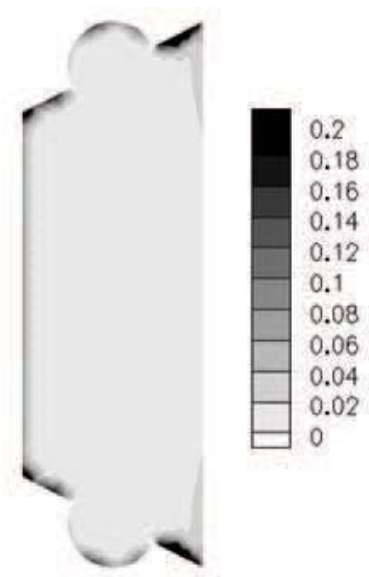

2 weeks

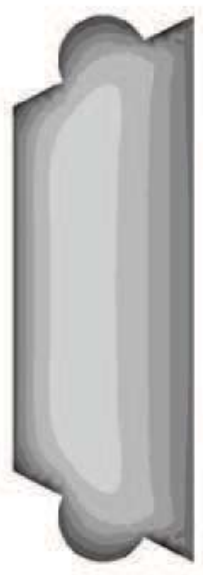

3 months

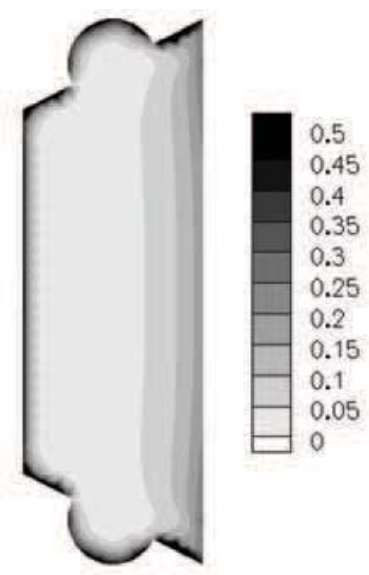

1 month

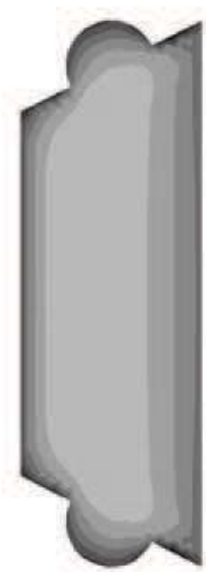

4 months

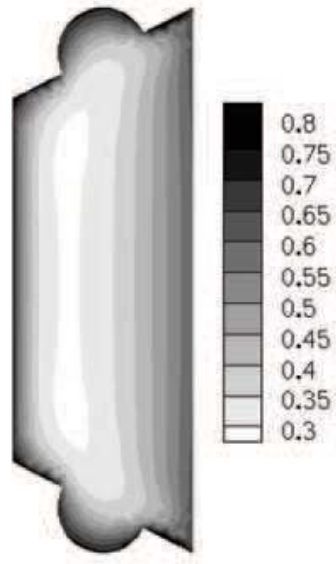

2 months

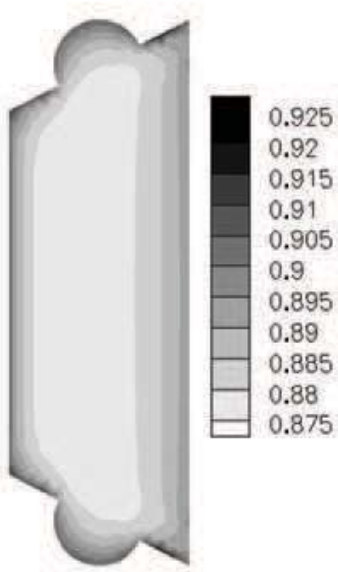

6 months

Figure 11. Temporal evolution of the volume fraction of lamellar bone around an implant with a high surface microtopography.

early deposition of bone inside the grooves, characteristic of rough implants. From Fig. 11 we conclude that the grooves have a stimulating effect on bone formation, that can be observed after 1 and 2 months of healing, when the volume fraction of lamellar bone is greater inside the grooves compared with the rest of the cavity. In the case of the polished implant, this effect also appears after the second month of healing, but its magnitude is lower, as could be expected. The temporal evolution of the global level of bone formation is similar to the results obtained in Part 1 , that were within the range of experimental 
results.
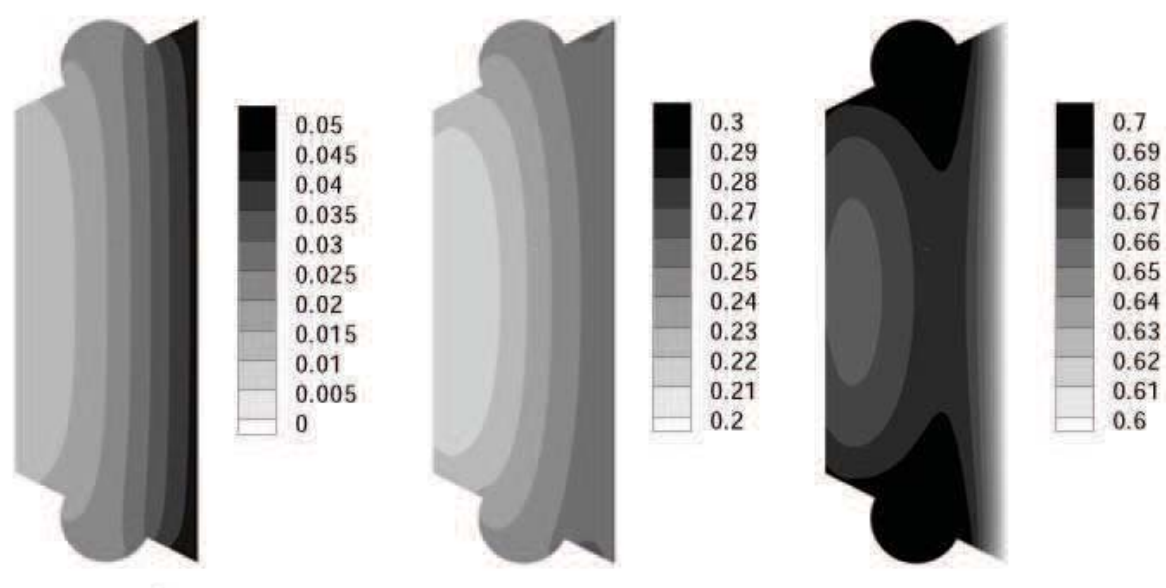

2 weeks
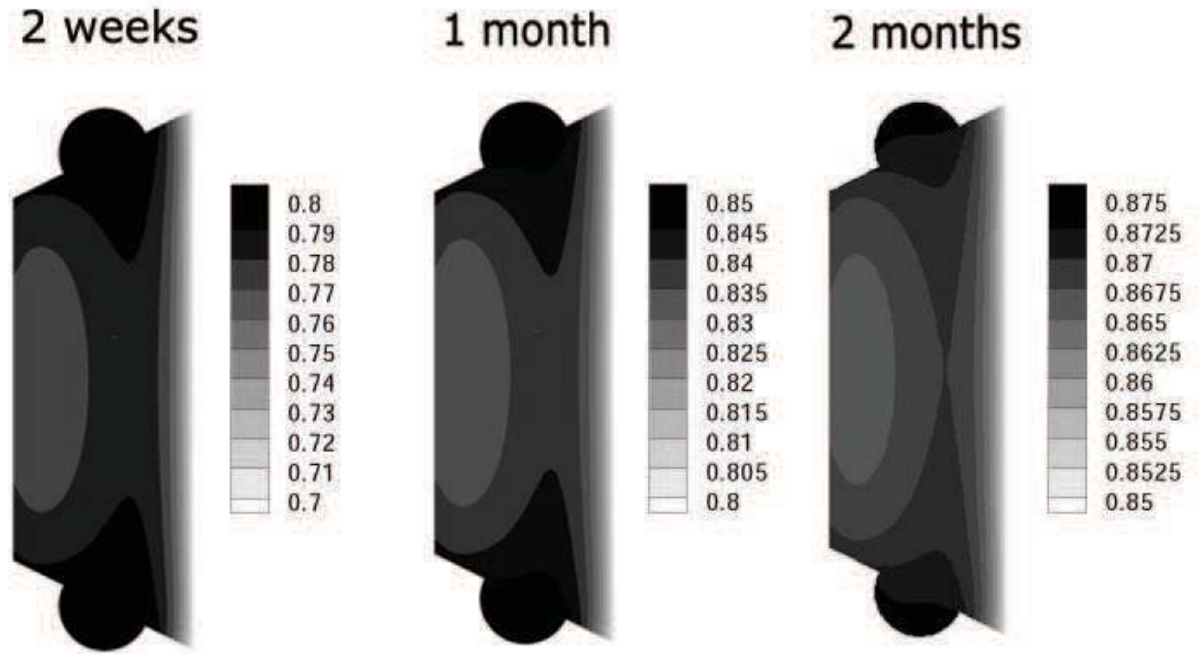

3 months

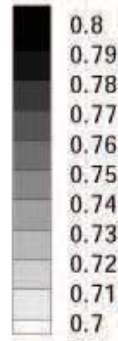

4 months

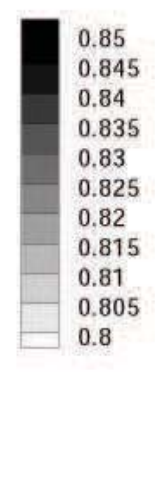

\section{6 months}

Figure 12. Temporal evolution of the volume fraction of lamellar bone around an implant with a low surface microtopography.

\section{Discussion}

In Part 1 of this work (Moreo et al., 2008) a mathematical model for the simulation of bone healing around endosseous implants was presented, its biological basis was discussed and numerical simulations were performed to show some of its capabilities. The goal of this second part was to further evaluate the model 
by means of analysing the effect that certain factors (initial cell population at the surface of the host bone, implant geometry and cell stimulation) have upon peri-implant bone ingrowth and studying how the composition of the matrix is altered as the ossification front propagates. This goal has been achieved through the combination of theoretical analysis and numerical simulations.

In Section 3, a mathematical analysis of the model permits to address the effect of cell stimulation on osteoconduction. The main biological conclusion that can be drawn from this analysis is that the speed of propagation of the osteogenic cells front, as well as the level of osteoblast concentration that can be achieved at the end of the osteoconduction phase, depend on the degree of stimulation of the cells and initial density of osteogenic cells at the surface of the host bone. For instance, an initial high concentration of osteogenic cells or growth factors at the surface of the host bone, typical of trabecular bone, does not guarantee a fast invasion of the cavity between bone and implant by cells if they lie under very reduced cell stimulation (regime I). However, the migration of cells will be fostered if they are sufficiently stimulated. In addition, in this second case (regimes II and III), the density of osteogenic cells at the surface of the bone does play an important role, since the final state achieved at the end of osteoconduction as well as the speed at which this state is reached depend on the initial number of cells. This varying density may be related to the type of bone. Trabecular bone, although distinguishable by their inferior mechanical properties compared to cortical bone, is a rich source of osteogenic cells, that are derived from both the endosteal trabecular surfaces and the marrow that fills the pores of the bone tissue. Therefore, assuming that the density of trabecular bone where an implant is going to be placed is high enough to provide sufficient mechanical stability, peri-implant bone 
healing may be able to evolve rapidly compared to a scenario in which the macroarchitecture of the host bone is cortical. This was described by Davies (2003) as "the paradox of poor quality bone". Nevertheless, note that a region of trabecular bone with excessive low density and poor mechanical properties is not adequate for the placement of an implant since its primary stability is compromised, what can lead to the occurrence of excessive micromotions and the development of a fibrous capsule. This is usually the origin of higher failure rates of dental implants in the posterior maxilla (Palmer, 1999).

Along the paper, $\chi$ has been always considered as a measure of the general level of cell stimulation, without making explicit reference to the specific stimuli that are comprised in this parameter. The mechanical environment, for example, can be one possible origin of cell stimulation. In fact, it is known that moderate mechanical loading stimulates two crucial phenomena in periimplant bone healing: osteogenic cell differentiation (Carter and Giori, 1991; Palma et al., 2005; Ignatius et al., 2005), quantified by $\alpha_{m b}$ in our model, and growth factor secretion (Raab-Cullen et al., 1994; Yeh et al., 1994; Kobayashi et al., 2000), denoted by $\alpha_{2}$. According to these experimental results, an hypothetical rise of the level of mechanical stimulation would lead to an increase of $\chi$ and, thus, $\chi$ could be considered to some extent as a measure of mechanical stimulation (see Fig. 13).

However, the previous reasoning is only valid under low or moderate levels of mechanical loading, since under severe mechanical solicitations phenomena such as fibrous tissue formation could take place, what has not been taken into account in the model. In particular, $\chi$ would increase with the mechanical stimulation, due to the increase of $\alpha_{m b}$ and $\alpha_{2}$, but would decrease under severe loading (Fig. 13), due to an increase of $A_{b}$ (osteoblast die under ex- 


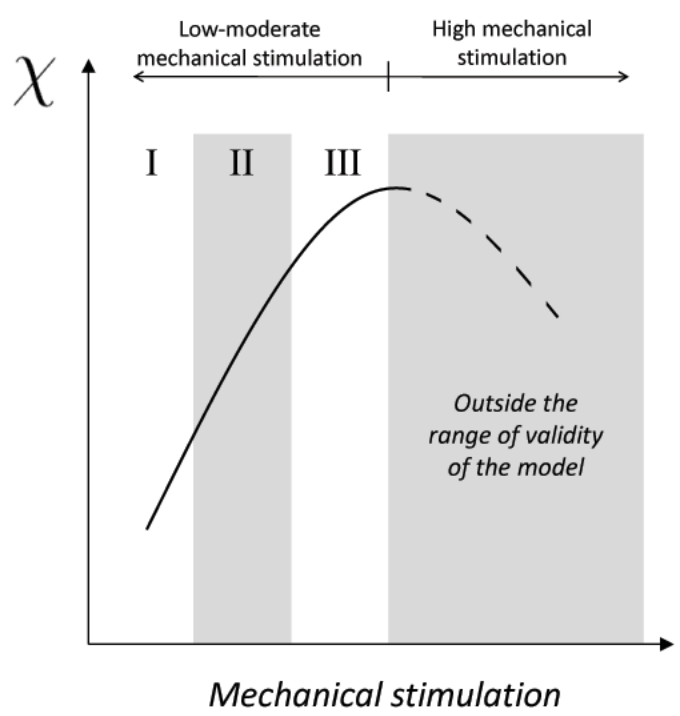

Figure 13. Qualitative dependence of $\chi$ on the level of mechanical stimulation. The model is only valid in the low-moderate loading case and must be considered just as a rough approximation in the case of severe loading. The three different regimes (I, II, III) of Fig. 1 are shown.

cessive load) and a decrease of $\alpha_{m b}$ (osteogenic cells do not differentiate into osteoblasts under these conditions, but into chondrocytes or fibroblasts). So, one possible specific interpretation of $\chi$, with the limitations previously mentioned, is the level of mechanical cellular stimulation. Although the present model and analysis is only valid for low and moderate mechanical stimulation of the implant, this situation is the most interesting one, given that scenarios of excessive mechanical loads are a priori known to be harmful for the osseointegration of an implant and must be avoided.

Furthermore, an increase of $\chi$ can effectively be the result of mechanical stimulation, but it can also be the consequence of any other sort of adequate cell stimulation. For example, controlled release of osteogenic growth factors from the surface of the implant.

Additionally, we examined the effect that the geometry of an implant can exert 
on the deposition of bone around it. In particular, we decided to find out if the model was able to explain the early formation of bone that has been observed experimentally inside macroscopic grooves located at the surface of the threads of dental implants (Hall et al., 2005). By means of numerical simulations we show that the model indeed predicted this preferential formation of new bone in the grooves in agreement with the experimental results and offer a simple explanation: the shape of the grooves promotes the concentration inside of them of growth factors and signalling molecules secreted by platelets, so they become a primary site of migration for osteogenic cells where additionally their differentiation into osteoblasts and the laying down of bone matrix are fostered. This opens a new line of research focused on the study of the influence of the shape of the threads of dental implants on the biological processes of bone healing.

To conclude, we highlight the two main original aspects of this second part of work:

- The study of the influence of the generic level of cell stimulation on periimplant bone healing. It is remarkable that this study has been done analytically, what has permitted to acquire information regarding the qualitative behaviour of the model for all the values of the model parameters. This is useful given the complexity of the model and the large number of parameters.

- The study of the effect of the grooves placed at the surface of the implant threads upon the biology of peri-implant bone healing. The majority of previous theoretical works on this point have looked into the influence of the geometry of the threads on the mechanical behaviour of the implant, not taking into account any biological implication. 
The model must be nonetheless improved to accommodate biological phenomena such as endochondral ossification or vasculogenesis to augment its range of application. The explicit consideration of the mechanical behaviour of the host bone and the implant is also of interest, since it would allow to study other important issues that influence the performance of endosseous implants, such as primary stability or the mechanical condition of the surrounding bone.

\section{Acknowledgments}

The authors gratefully acknowledge the research support of the Spanish Ministry of Health through the CIBER-BBN and the Spanish Ministry of Science and Technology through Research Projects DPI2006-14669 and DPI200609692 and the FPU graduate research fellowship program.

\section{References}

Bailón-Plaza, A., van der Meulen, M. C. H., 2001. A mathematical framework to study the effects of growth factor influences on fracture healing. J. Theor. Biol. 212, 191-209.

Berglundh, T., Abrahamsson, I., Lang, N. P., 2003. De novo alveolar bone formation adjacent to endosseous implants. A model study in the dog. Clin. Oral Impl. Res. 14, 251-262.

Carter, D. R., Giori, N. J., 1991. Effect of mechanical stress on tissue differentiation in the bony implant bed. In: The bone-biomaterial interface. Toronto, University of Toronto Press, p. 367. 
Dale, P. D., Olsen, L., Maini, P. K., Sherratt, J. A., 1995. Travelling waves in wound healing. Forma 10, 205-222.

Davies, J. E., 2003. Understanding peri-implant endosseous healing. J. Dental Educ. 67, 932-949.

Dimitriou, R., Tsiridis, E., Giannoudis, P. V., 2005. Current concepts of molecular aspects of bone healing. Injury, Int. J. Care Injured 36, 1392-1404.

Ganong, W. F., 2005. Review of medical physiology, 22nd Edition. McGrawHill.

Hall, J., Miranda-Burgos, P., Sennerby, L., 2005. Stimulation of directed bone growth at oxidized titanium implants by macroscopic grooves; an in vivo study. Clin. Implant Dent. Relat. Res. 7, S76-82.

Ignatius, A., Blessing, H., Liedert, A., Schimdt, C., Neidlinger-Wilke, C., Kaspar, D., Friemert, B., Claes, L., 2005. Tissue engineering of bone: effects of mechanical strain on osteoblastic cells in type I collagen matrices. Biomaterials $26,311-318$.

Joyce, M. E., Terek, R. M., Jingushi, S., Bolander, M. E., 1990. Role of transforming growth factor- $\beta$ in fracture repair. Ann. N. Y. Acad. Sci. 593, $107-$ 123.

Kark, L. R., Karp, J. M., Davies, J. E., 2006. Platelet releasate increases the proliferation and migration of bone marrow-derived cells cultured under osteogenic conditions. Clin. Oral Impl. Res. 17, 321-327.

Kikuchi, L., Park, J. Y., Victor, C., Davies, J. E., 2005. Platelet interactions with calcium-phospate-coated surfaces. Biomaterials 26, 5285-5295.

Kobayashi, Y., Hashimoto, F., Miyamoto, H., et al., 2000. Force-induced osteoclast apoptosis in vivo is accompanied by elevation in transforming growth factor $\beta$ and osteprotegerin expression. J. Bone Miner. Res. 15, 1924-1934.

Linkhart, T. A., Mohan, S., Baylink, D. J., 1996. Growth factors for bone 
growth and repair: IGF, TGF $\beta$ and BMP. Bone 19, 1S-12S.

Moreo, P., García-Aznar, J. M., Doblaré, M., 2008. Bone ingrowth on the surface of endosseous implants. Part 1: Mathematical model. J. Theor. Biol. submitted.

Murray, J. D., 2005. Mathematical Biology, 3rd Edition. Springer.

Olsen, L., Sherratt, J. A., Maini, P. K., 1996. A mathematical model for fibroproliferative wound healing disorders. Bull. Math. Biol. 58, 787-808.

Palma, F. D., Guignandon, A., Chamson, A., Lafage-Proust, M. H., Laroche, N., Peyroche, S., Vico, L., Rattner, A., 2005. Modulation of the responses of human osteoblast-like cells to physiologic mechanical strains by biomaterial surfaces. Biomaterials 26, 4249-4257.

Palmer, R., 1999. Introduction to dental implants. Brit. Dental J. 187, 127132.

Park, J. Y., Gemmell, C. H., Davies, J. E., 2001. Platelet interactions with titanium: modulation of platelet activity by surface topography. Biomaterials $22,2671-2682$.

Raab-Cullen, D. M., Thide, M. A., Petersen, D. N., Kimmel, D. B., Recker, R. R., 1994. Mechanical loading stimulates rapid changes in periosteal gene expression. Calcif. Tissue Int. 55, 473-478.

Sela, M. N., Badihi, L., Rosen, G., Steinberg, D., Kohavi, D., 2007. Adsorption of human plasma proteins to modified titanium surfaces. Clin. Oral Implants Res. 18, 630-638.

Strogatz, S. H., 2001. Nonlinear dynamics and chaos, 1st Edition. Perseus Books Group.

Vandamme, K., Naert, I., Geris, L., Sloten, J. V., Puers, R., Duyck, J., 2007. Influence of controlled immediate loading and implant design on periimplant bone formation. J. Clin. Periodontol. 34, 172-181. 
Yeh, J. K., Aloia, J. F., Chen, M., Sprintz, S., 1994. Effect of growth hormone administration and treadmill exercise on the body composition of rats. J. Appl. Physiol. 77, 23-29.

\section{A Description of the full model equations}

Below we present the equations that particularise the evolution of each model variable corresponding to (Moreo et al., 2008):

Platelets, $c(\mathbf{x}, t)$

$$
\frac{\partial c}{\partial t}=\nabla \cdot\left[D_{c} \nabla c-H_{c} c \nabla p\right]-A_{c} c
$$

$D_{c}$ is a diffusion coefficient that quantifies the rate of cell random dispersal, the extent of platelet adhesion to the surface depends on the value of the parameter $H_{c}$ and $A_{c}$ is the rate of cell removal due inflammatory mechanisms.

Osteogenic CELls, $m(\mathbf{x}, t)$

$$
\begin{aligned}
\frac{\partial m}{\partial t} & =\nabla \cdot\left[D_{m} \nabla m-m\left(B_{m 1} \nabla s_{1}+B_{m 2} \nabla s_{2}\right)\right] \\
& +\underbrace{\left(\alpha_{m 0}+\frac{\alpha_{m} s_{1}}{\beta_{m}+s_{1}}+\frac{\alpha_{m} s_{2}}{\beta_{m}+s_{2}}\right) m\left(1-\frac{m}{N}\right)}_{\text {Proliferation }}-\underbrace{\frac{\alpha_{m b} s_{1}}{\beta_{m b}+s_{1}} m}_{\text {Differentiation }}-\underbrace{A_{m} m}_{\text {Death }}
\end{aligned}
$$

Osteogenic cell flux comes from random cell movement that can be biased by the presence of growth factors. Mathematically this is modelled by means of linear diffusion, with coefficient $D_{m}$, and linear chemotaxis along gradients of 
the growth factors $s_{1}$ and $s_{2}$ with coefficients $B_{m 1}$ and $B_{m 2}$. For the kinetics, there is a proliferative term consisting of a logistic growth with a natural linear rate $\alpha_{m 0}$ that can be enhanced in a dose-dependent manner by the presence of $s_{1}$ and $s_{2}$; phenotypic differentiation into osteoblasts is stimulated by growth factor $s_{1}$; and natural cell death is assumed to take place at a linear rate $A_{m}$.

Osteoblasts, $b(\mathbf{x}, t)$

$$
\frac{\partial b}{\partial t}=\frac{\alpha_{m b} s_{1}}{\beta_{m b}+s_{1}} m-A_{b} b
$$

The kinetics has a source term of differentiation from the osteogenic phenotype at a maximum rate $\alpha_{m b}$ and a decay term representing differentiation into osteocytes with rate $A_{b}$.

GENERIC GROWTH FACTOR $1, s_{1}(\mathbf{x}, t)$

$$
\frac{\partial s_{1}}{\partial t}=\nabla \cdot\left[D_{s 1} \nabla s_{1}\right]+\left(\frac{\alpha_{c 1} p}{\beta_{c 1}+p}+\frac{\alpha_{c 2} s_{1}}{\beta_{c 2}+s_{1}}\right) c-A_{s 1} s_{1}
$$

Random dispersal of the growth factor is modelled as a linear diffusion with coefficient $D_{s 1}$. The first kinetic term takes into account the secretion of $s_{1}$ by platelets, which is fostered by the concentration of adsorbed proteins $p$, whose effect is quantified by $\alpha_{c 1}$, and the own growth factor $s_{1}$, quantified by $\alpha_{c 2}$. There is also a natural decay of the growth factor with rate $A_{s 1}$.

GeneriC GROWTH FACTOR $2, s_{2}(\mathbf{x}, t)$

$$
\frac{\partial s_{2}}{\partial t}=\nabla \cdot\left[D_{s 2} \nabla s_{2}\right]+\frac{\alpha_{m 2} s_{2}}{\beta_{m 2}+s_{2}} m+\frac{\alpha_{b 2} s_{2}}{\beta_{b 2}+s_{2}} b-A_{s 2} s_{2}
$$


The structure of the equation is completely equivalent to the one of $s_{1}$, although in this case there are two source terms corresponding to secretion of $s_{2}$ by osteogenic cells and osteoblasts.

Fibrin NETWORK VOLUME FRACTION, $v_{f}(\mathbf{x}, t)$

$$
\frac{\partial v_{f}}{\partial t}=-\frac{\alpha_{w} s_{2}}{\beta_{w}+s_{2}} b v_{f}\left(1-v_{w}\right)
$$

The only kinetic term comes from partial substitution of the fibrin network by woven bone matrix, that is, secretion of new bone by osteoblasts. The rate of deposition of woven bone depends on the value of the parameter $\alpha_{w}$, is stimulated by the presence of $s_{2}$ and increases linearly with the number of osteoblasts.

WOVEN BONE VOLUME FRACTION, $v_{w}(\mathbf{x}, t)$

$$
\frac{\partial v_{w}}{\partial t}=\frac{\alpha_{w} s_{2}}{\beta_{w}+s_{2}} b v_{f}\left(1-v_{w}\right)-\gamma v_{w}\left(1-v_{l}\right)
$$

Woven bone formation is taken into account by the first term, that was explained in the previous paragraph and describes the formation of woven bone that replaces the initial fibrin network. The second term quantifies the remodelling of woven bone into lamellar bone, being $\gamma$ the linear rate at which this process takes place.

LAMELLAR BONE VOLUME FRACTION, $v_{l}(\mathbf{x}, t)$

$$
\frac{\partial v_{l}}{\partial t}=\gamma v_{w}\left(1-v_{l}\right)
$$


Finally, the only contribution to the evolution of lamellar bone comes from remodelling of woven bone.

\section{B Steady states and bifurcations}

Expressions for $\mathbf{z}_{t}$ and $\mathbf{z}_{0}$ are trivial to obtain and were presented in Section 3.2. Analytical expressions for $\mathbf{z}_{ \pm}$are, however, not quite as simple and are obtained by searching nontrivial solutions of $f=g=h=0$. In particular, $s_{2 \pm}$ are given by the real positive roots of the polynomial $a_{2} s_{2}^{2}+a_{1} s_{2}+a_{0}$, where coefficients $a_{i}$ are defined as

$$
\begin{aligned}
& a_{2}=A_{s 2}\left(1+\alpha_{m}\right) \\
& a_{1}=\left(1+\alpha_{m}\right)\left(\beta_{2} A_{s 2}-\chi m_{0}\right)+\alpha_{m} \chi\left(m_{0}-1\right)+\beta_{m} A_{s 2} \\
& a_{0}=\beta_{m}\left(\beta_{2} A_{s 2}-\chi m_{0}\right)
\end{aligned}
$$

Once $s_{2 \pm}$ are determined, $m_{ \pm}$and $b_{ \pm}$are straightforwardly derived from the condition $g=h=0$ :

$$
\begin{aligned}
m_{ \pm} & =A_{s 2} \chi^{-1}\left(\beta_{2}+s_{2 \pm}\right) \\
b_{ \pm} & =\alpha_{m b} A_{b}^{-1} m_{ \pm}
\end{aligned}
$$

Taking into account that $a_{2}>0$, the existence conditions of the steady states $\mathbf{z}_{ \pm}$can be summarised as follows:

- If $a_{1}<0, a_{0}>0$ and $a_{1}^{2}-4 a_{2} a_{0}>0$, then $\mathbf{z}_{+}$and $\mathbf{z}_{-}$exist, both with real positive values (Fig. 1b). The sign of $a_{1}$ and $a_{0}$ is derived from Descartes' 
rule of signs and the positiveness of the determinant is required to ensure the obtention of real solutions.

- If $a_{0}<0$, then the determinant $a_{1}^{2}-4 a_{2} a_{0}$ is always positive, so the solutions are real. However, the product of the solutions verifies in this case $s_{+} s_{-}=a_{0} a_{2}^{-1}<0$, indicating that we only have one positive solution. We are therefore in the situation of Fig. 1c.

- Otherwise, there are no such steady states.

The stability of the steady states can be determined in the standard way from the study of the eigenvalues of the jacobian matrix of the system and the main results have been presented in Section 3.2.

¿From the existence conditions it is easy to deduce the bifurcation values of the system. In first place, the saddle-point bifurcation leading to the appearance of the pair of steady states $\mathbf{z}_{ \pm}$clearly occurs when $a_{1}^{2}=4 a_{2} a_{0}, a_{1}<0, a_{0}>0$. From this condition the value of $\chi^{(1)}$ is obtained as

$$
\chi^{(1)}=\frac{-a_{1}^{\prime}+\sqrt{a_{1}^{\prime 2}-4 a_{2}^{\prime} a_{0}^{\prime}}}{2 a_{2}^{\prime}}
$$

where coefficients $a_{i}^{\prime}$ are defined as

$$
\begin{aligned}
& a_{2}^{\prime}=\left(\alpha_{m}+m_{0}\right)^{2} \\
& a_{1}^{\prime}=4 m_{0} A_{s 2}\left(1+\alpha_{m}\right) \beta_{m}-2 A_{s 2}\left(\alpha_{m}+m_{0}\right)\left[\beta_{m}+\beta_{2}\left(1+\alpha_{m}\right)\right] \\
& a_{0}^{\prime}=A_{s 2}^{2}\left[\beta_{m}-\beta_{2}\left(1+\alpha_{m}\right)\right]^{2}
\end{aligned}
$$

Finally, the transcritical bifurcation happens when $a_{0}=0$, leading to the following expression for $\chi^{(2)}$ : 


$$
\chi^{(2)}=\beta_{2} A_{s 2} m_{0}^{-1}
$$

\section{Travelling wave solutions}

Travelling wave solutions are investigated by means of the variable transformations $m(x, t)=M(r), s_{2}(x, t)=S_{2}(r)$ and $b(x, t)=B(r)$, where $r=x$-at and $a$ is the wave velocity. This change of variables yields a system of five coupled ordinary differential equations for $M, S_{2}, B, U=d N / d z$ and $V=d S_{2} / d z$ in terms of the travelling wave coordinate, $r$. We denote the chronic non healing steady state in the $\left(M, S_{2}, B, U, V\right)$-space by $\mathbf{Z}_{t}=(0,0,0,0,0)$, the low density state by $\mathbf{Z}_{0}=\left(m_{0}, 0, b_{0}, 0,0\right)$ and the high density states by $\mathbf{Z}_{ \pm}=\left(m_{ \pm}, s_{2 \pm}, b_{ \pm}, 0,0\right)$

A necessary condition for travelling wave transitions from $\mathbf{Z}_{t}$ to $\mathbf{Z}_{0}$ is the existence of a heteroclinic trajectory (Strogatz, 2001) from $\mathbf{Z}_{t}$ to $\mathbf{Z}_{0}$ in the $\left(M, S_{2}, B, U, V\right)$-space. The existence of this trajectory can be intuitively expected from the look of the phase portrait of Fig. 1 and the shape of Eqs. (1) when we set $s_{2}=0$. Additionally we must require that this trajectory lies entirely within the subspace $\left(M, S_{2}, B\right) \geq(0,0,0)$ in order to be physically realistic. Thus, the eigenvalues of the jacobian matrix of the system for both equilibrium points must be real to avoid locally oscillatory solutions that would cause $M, S_{2}$ and $B$ to be negative for some $r$. A linear stability analysis at $\mathbf{Z}_{t}$ and $\mathbf{Z}_{0}$ shows that this condition is satisfied if and only if $a \geq a_{\text {min }}^{(1)}=$ $2 \sqrt{D_{m}\left[\sigma-\left(\alpha_{m b}+A_{m}\right)\right]}$ when $\chi \in\left(0, \chi^{(2)}\right)$ and $a \geq \max \left\{a_{\min }^{(1)}, a_{\min }^{(2)}\right\}$ when 
$\chi \in\left(\chi^{(2)}, \infty\right)$, where

$$
a_{\text {min }}^{(2)}=2 \sqrt{D_{s}\left(\frac{\chi m_{0}}{\beta_{2}}-A_{s 2}\right)}
$$

The rigorous proof of existence of a heteroclinic trajectory from $\mathbf{Z}_{0}$ to $\mathbf{Z}_{+}$is a very hard task, but linear stability analysis reveals the existence of a stable local manifold at $\mathbf{Z}_{0}$ and an unstable manifold at $\mathbf{Z}_{+}$. Therefore, with the conjecture that such heteroclinic trajectory exists, travelling wave solutions are possible. The condition $\left(M, S_{2}, B\right) \geq(0,0,0)$ does not impose any lower bound on the wave speed when $\chi \in\left(\chi^{(1)}, \chi^{(2)}\right)$, but the restriction $a \geq a_{\text {min }}^{(2)}$ is obtained when $\chi \in\left(\chi^{(2)}, \infty\right)$. Numerical solution of the system confirms that this trajectory does exist when $\chi \in\left(\chi^{(1)}, \infty\right)$.

The same argument applies for the case of a transition between $\mathbf{Z}_{t}$ and $\mathbf{Z}_{+}$. Numerical solution of the system demonstrates that this travelling wave exists only when $\chi \in\left(\chi^{(2)}, \infty\right)$. In this case the lower bound that is obtained for the wave speed is $a_{m i n}^{(1)}$. 